\title{
DINÂMICAS QUE ENSINAM: A METODOLOGIA DE PROJETO NO ENSINO DE ARQUITETURA'
}

\author{
Lessons learned from lessons given \\ and architectural design methods
}

\section{Daniel de Carvalho Moreira', Doris Catharine Cornelie Knatz Kowaltowski', Renata Maria Geraldini Beltramin'}

RESUMO Este trabalho apresenta várias dinâmicas de ensino dentro de disciplinas de graduação e pós-graduação da Faculdade de Engenharia Civil, Arquitetura e Urbanismo (FEC) da Universidade Estadual de Campinas (Unicamp). As experiências tocaram em questões relacionadas ao processo de projeto em arquitetura. O processo de projeto é entendido como um ciclo de atividades que compreende a concepção, a descrição, a construção e o uso do espaço construído. É dada ênfase às reflexões sobre o programa arquitetônico e às possíveis dinâmicas para a fase analítica do projeto. Dinâmicas são consideradas importantes, pois estimulam novos pontos de vista e oferecem alternativas ao projetista na resolução de problemas. No ensino, elas podem surpreender alunos e professores, além de estimular novos nichos de pesquisa acadêmica. A relação ensino-pesquisa no ensino superior é discutida. São apresentadas experiências de disciplinas distintas de graduação e pós-graduação. Um curso de curta duração contribuiu também com outras lições e dinâmicas. O propósito do presente artigo é apresentar as dinâmicas aplicadas, independentemente da situação original em que foram ministradas, e discutir as contribuições e resultados obtidos nessas experiências, especialmente em relação às pedagogias específicas em metodologia de projeto em arquitetura.

PALAVRAS-CHAVE: Ensino de projeto, Dinâmicas de ensino, Processo de projeto.

ABSTRACT This paper presents several teaching experiences of disciplines in the undergraduate and graduate courses of the School of Civil Engineering, Architecture and Urban Design (FEC) of the State University of Campinas (Unicamp). The teaching examples discuss some of the issues of the contemporary design process in architecture. The design process is seen as a cycle of activities that include design, documentation, construction and use of built spaces. Emphasis is given to the architectural programming phase of this process and possible dynamics and activities for this analytical phase of this process. Dynamics are considered important, enabling designers to leave their comfort zone to experiment new points of view. In education, they may astonish students and faculty, stimulating as well new types of academic research. The research-teaching relationship is discussed at the higher education level. Examples Experiences of distinct undergraduate and graduate disciplines are presented. The teaching experience of a mini course provided further lessons learned. The purpose of this paper is to present the teaching experiences, regardless of the original situation in which they were applied, and discuss the contributions and results obtained from these examples, especially in the light of appropriate pedagogies for courses on design methods in architecture.

KEYWORDS: Design education, Teaching dynamics, Design process.

\section{How to cite this article:}

MOREIRA, D. C.; KOWALTOWSKI, D. C. C. K.; BELTRAMIN, R. M. G. Dinâmicas que ensinam: a metodologia de projeto no ensino de arquitetura. Gestão e Tecnologia de Projetos, São Carlos, v. 11, n. 1, p. 55-69, jan./jun. 2016 http://dx.doi.org/10.11606/gtp.v11i1.99197

Fonte de financiamento: Declaram não haver. Conflito de interesse: Declaram não haver. Submetido em: 19 jun. 2015 Aceito em: 17 dez. 2015 


\section{INTRODUÇÃO}

O processo de projeto é entendido como um ciclo de atividades que compreende a concepção, a descrição, a construção e o uso do espaço construído. Partindo da definição da arquitetura como a ação, a arte ou a atividade de organizar espaços adequados às funções e à permanência humana com qualidade (UNWIN, 1997), é possível caracterizar o processo de projeto enquanto um conjunto de atividades que visa à obtenção de uma solução arquitetônica a um ou mais problemas relativos à organização e à adequação de um determinado espaço a necessidades humanas específicas. O próprio termo "processo de projeto" destaca a fase de proposição da forma diminuindo a presença de outras etapas. Muitas vezes a expectativa pelo momento da solução ofusca outras conquistas envolvidas no processo como, por exemplo, compreender o problema. Dessa maneira, rever algumas certezas que direcionam os modos de uso dos edifícios e dos espaços urbanos possibilita um envolvimento renovado e apaixonado com as mudanças que a boa arquitetura pode operar. As inovações arquitetônicas não dependem apenas de formas inusitadas, mas podem surpreender ao propor novos modos de viver. Para discutir e aprofundar o entendimento desse processo enriquecido de projeto, algumas experiências de ensino são apresentadas nesse artigo. No ensino, é dada ênfase às reflexões sobre o programa arquitetônico e às dinâmicas propostas para essa fase analítica do processo de projeto. Considera-se que dinâmicas podem tirar o projetista da zona de conforto ao oferecer novos pontos de vista sobre o problema de projeto. No ensino, elas podem surpreender alunos e professores, além de estimular novos nichos de pesquisa acadêmica. São esses os argumentos que o presente trabalho propõe discutir, bem como apresentar a aplicabilidade e a contribuição das dinâmicas descritas. A base das dinâmicas consiste, principalmente, na exploração da literatura sobre metodologia de projeto e psicologia ambiental em arquitetura.

Nos últimos cinco anos, docentes da Universidade Estadual de Campinas (Unicamp) dedicaram-se aos questionamentos sobre o processo de projeto e às possibilidades de criar metodologias e ferramentas para dar suporte às suas fases de reflexão. Nesse contexto, foi criado o programa de pósgraduação da Faculdade de Engenharia Civil, Arquitetura e Urbanismo da Unicamp em Arquitetura, Tecnologia e Cidade (ATC), credenciado pela CAPES em 2011, saindo de uma área de concentração já consolidada, com linhas de pesquisa como: teoria e metodologia de projeto, conforto ambiental e tecnologia e gestão do ambiente construído. Dentro desse novo programa de pós-graduação, foram concebidos novos meios de ensino, os quais foram aplicados em diversos contextos, sobretudo em disciplinas de projeto em cursos de graduação e de extensão. As dinâmicas de ensino apresentadas neste trabalho foram elaboradas com o objetivo de ampliar o debate sobre os métodos de projeto e estimular novos estudos. Um minicurso foi oferecido durante o Encontro Nacional sobre Conforto no Ambiente Construído (ENCAC) 2013 em Brasília para um público variado, composto por professores, alunos e pesquisadores. Outras dinâmicas foram incluídas nesse trabalho, tais como: a aplicação do sistema Hierarchical Decomposition of Systems (HIDECS) para a elaboração de requisitos de projeto, métodos de estímulo à criatividade, escala semântica de valores e jogo de cartas (sendo o tema desta última dinâmica, a arquitetura escolar). A tipologia arquitetônica "bibliotecas" também foi delineada com a criação de parâmetros de projeto que podem ser aplicados para avaliações pré e pósprojeto. Em outro estudo programado, na forma de workshop ministrado no curso de mestrado profissionalizante na UFRN, algumas das atividades e dinâmicas foram testadas. Diante desse contexto, este artigo apresenta não somente o relato das experiências didáticas e suas respectivas e resultantes lições aprendidas como também uma reflexão sobre o ensino de projeto arquitetônico e as pesquisas realizadas na área de metodologia de projeto. 


\section{METODOLOGIA DE PROJETO ARQUITETÔNICO}

Durante a década de 1950, arquitetos e engenheiros atentos ao panorama científico procuravam aplicar novas técnicas ao desenvolvimento do projeto para melhorar a qualidade do processo e dos seus produtos. Reunir experiências e teorias na aplicação de técnicas sistemáticas de projeto, influenciadas por novas práticas científicas, como a Teoria dos Sistemas, Teoria da Informação e a Pesquisa Operacional foram os objetivos desses primeiros estudos sobre metodologia de projeto em arquitetura (BAYAZIT, 2004; BROADBENT; WARD, 1969; BUCHANAN, 1992; CROSS, 1984; KOWALTOWSKI, 1992; KOWALTOWSKI et al., 2006; MOREIRA; KOWALTOWSKI, 2009). No Brasil, os Design Methods não tiveram expressiva repercussão na atividade profissional dos escritórios de projeto e influenciaram pouco os programas de ensino ou pesquisa das escolas de engenharia e arquitetura (CELANI, 2003). Como consequência, observa-se uma falta de consenso na definição de uma estrutura do processo de projeto e o procedimento de tentativa e erro na concepção do edifício é perpetuado.

Quarenta anos após o início do movimento dos Design Methods, vários autores distinguem diferentes fases que caracterizaram sua evolução (BAYAZIT, 2004; CROSS, 1984; VAN DER VOORDT; VAN WEGEN, 2005), sendo a primeira subdivisão em fases identificada por Horst Rittel, logo no começo dos anos de 1970, como a "primeira geração dos métodos de projeto" (RITTEL, 1973). Os métodos propostos por essa geração se caracterizavam pela sistematização de um processo de três fases - a análise, a síntese e a avaliação - e eram baseadas, principalmente, nas técnicas de pesquisa operacional. São conhecidos também como "métodos sistemáticos de projeto”. Ao identificar a primeira geração dos métodos de projeto, Rittel propôs uma segunda geração, que se caracterizava por considerar, nas decisões de projeto, o envolvimento do usuário e os seus objetivos (BAYAZIT, 2004). Na década de 1980, a abordagem dos Design Methods mudou novamente, e o projeto passou a ser visto como uma ciência específica que não precisava mais procurar argumentos nos princípios da filosofia da ciência (CROSS, 2006).

Durante seu desenvolvimento, os Design Methods repercutiram em áreas diversas e deram origem a importantes contribuições, como a Avaliação Pós-Ocupação (PREISER et al., 1990; ORNSTEIN; ROMERO, 1992), o programa arquitetônico, o Design Thinking, a inteligência artificial e a aplicação de técnicas computacionais para solucionar problemas de projeto e compor as formas dos objetos. Todas as transformações pelas quais os métodos de projeto passaram nos últimos anos contribuíram para estabelecer o assunto como uma disciplina independente, capaz de influenciar a própria ciência.

Os métodos de projeto em arquitetura discutem o processo de projeto e o apoio necessário para cada uma das suas fases com o objetivo de ampliar a produtividade dos projetistas e principalmente a qualidade dos seus produtos. Destaca-se nessa busca a fase analítica de elaboração do programa arquitetônico. Essa é uma das importantes fases que divide o contexto de um projeto arquitetônico em partes, procurando nelas os seus elementos principais (MOREIRA; KOWALTOWSKI, 2009). Essa divisão analítica pode ser chamada de estrutura do problema de projeto. $\mathrm{Na}$ sequência do processo, o desenvolvimento do projeto passa a resolver o problema colocado pelo programa. É por esse motivo que muitos autores consideram o procedimento de projeto uma atividade de síntese (CROSS, 2006; PEÑA; PARSHALL, 2001). Como a ciência envolve uma atividade analítica, os métodos de projeto que se baseavam na aplicação dos métodos científicos passaram a ser criticados por não considerar essa distinção entre cada um dos processos. Como consequência, estudos dos métodos de projeto se concentraram principalmente nas atividades de programação arquitetônica: a etapa de análise do processo de projeto. 
Segundo Kumlin (1995), o programa arquitetônico se estabeleceu como uma disciplina distinta em 1966. Até o final da década de 1960, algumas outras publicações haviam tratado do programa arquitetônico. Dentre elas estava o título Problem Seeking: an architectural programming primer, que ainda hoje é reeditado em novas atualizações (PEÑA; PARSHALL, 2012). O método do Problem Seeking estrutura as informações de projeto que um programa de necessidades deve abranger e serve como um checklist para a atividade de programação (PEÑA; PARSHALL, 2001). Alguns arquitetos também são conhecidos por serem os precursores da prática de desenvolver um programa de necessidades detalhado como parte do processo de projeto, como o arquiteto Louis Kahn (DOGAN; ZIMRING, 2002; HERSHBERGER, 1999; ALEXANDER et al., 1977) e Richard Neutra (FRAMPTON, 2007; LAMPRECHT, 2009).

A compreensão do programa arquitetônico é bastante variada. Em um primeiro momento, o programa é reduzido a uma mera lista de espaços com uma quantificação de áreas que definem principalmente as questões de custo. Um projeto que se inicia a partir de uma simplificação dessa natureza ignora a riqueza de discussões oferecida pela fase de pré-projeto, nas quais se estabelecem prioridades e se compreende de modo profundo o problema de um projeto. Para evitar essa abordagem pouco científica, as técnicas de programa de necessidades foram criadas. Elas são tão variadas quanto as estruturas que descrevem um contexto. Entretanto, fundamentalmente, os resultados de diferentes programas sobre um mesmo contexto deveriam ser ao menos semelhantes. No ensino de projeto, essas técnicas devem ser aplicadas e testadas: principalmente na pós-graduação o debate da fase analítica do processo deve estimular novas técnicas, ferramentas, métodos e também dinâmicas próprias.

\section{METODOLOGIA DE ENSINO DE PÓS-GRADUAÇÃO}

O ensino de pós-graduação em arquitetura está intrinsicamente ligado ao movimento dos Design Methods, já que a maioria desses programas teve o seu início nos anos 1950 e 1960, principalmente nos Estados Unidos (ESHERICK et al., 1963). Esses primeiros programas tiveram como objetivo abraçar a pesquisa como premissa e evitar a mera continuação de cursos de graduação que formam o profissional arquiteto, urbanista ou paisagista. Melhorar o ensino da história da arquitetura desencadeou os primeiros cursos de pós-graduação em arquitetura. A formação de professores com um profundo conhecimento e base sólida em pesquisa era visto como essencial na época (AKÖZER, 2005). A pesquisa em arquitetura também deveria sustentar estudos que tocam em questões sociais e econômicas em relação aos impactos dos projetos produzidos pelos profissionais. $\mathrm{O}$ levantamento de fatos sobre a arquitetura que iam além da sua qualidade estética era outro objetivo da ampliação da pesquisa e da criação de programas de pós-graduação. Neste contexto, os objetivos desses novos programas não deveriam ser diferentes de outros programas das ciências exatas ou sociais: deveriam encorajar estudos profundos e aplicar métodos científicos para contribuírem com conhecimento novo e originalidade. Objetivava-se também a formação de futuros líderes nas suas áreas, capazes de desenvolver um pensamento criativo e independente. No Brasil, um dos primeiros programas de pós-graduação foi o da Faculdade de Arquitetura e Urbanismo da Universidade de São Paulo (FAU/USP), o qual foi criado em 1971 e seguiu as tendências mundiais da época no ensino de arquitetura e urbanismo.

Para atingir os seus objetivos, os programas de pós-graduação se estruturam em disciplinas, na maioria de natureza teórica, com base em bibliografias específicas, seminários e debates sobre conceitos e controvérsias. O ponto alto dessas atividades acadêmicas, tanto para 
os alunos como para os seus orientadores, é a dissertação ou a tese, desenvolvidas com base, também, nas disciplinas de metodologia de pesquisa. Em um estudo realizado em 2002, Brian Paltridge examinou essas disciplinas, bem como as dissertações e teses dela resultantes, e demonstrou que a variedade de tipo de teses existentes é muito maior do que a abordada nessas disciplinas. Outro estudo europeu identificou que o contexto local em que se desenvolvem os programas de doutorado da área de arquitetura é extremamente importante (AKÖZER, 2005). Tal estudo mostrou que a questão da organização da profissão (processo de projeto) é um tema fundamental e apontou que as mídias (desenho, maquetes e modelos, CAD e prototipagem rápida) devem ser estudadas. Avaliação e controle de qualidade devem fazer parte do conjunto de pesquisas, assim como a validação (análise de projeto, APO, simulações), de acordo com o mesmo estudo. Finalmente, o estudo considerou que um debate sobre questões epistemológicas em relação às pesquisas em design e arquitetura é indispensável (AKÖZER, 2005).

Os diferentes tipos de pesquisa apresentam desafios variados e fazem exigências muito específicas sobre os alunos e seus orientadores. 0 profissional arquiteto desenvolve o seu projeto com esforços criativos sem necessariamente explicar ou validar a proposta. A pesquisa de doutorado, no entanto, é diferente, exigindo transparência no processo e resultados validados. Sabe-se que atividades criativas realizadas em ateliê de projeto compreendem formas de atividade de pesquisa, principalmente na fase de programa, as quais incluem a experimentação e a análise de dados. Mas os resultados dessas pesquisas raramente são tão cuidadosamente estruturados ou metódicos como devem ser os processos das pesquisas científicas da área. O projetista está preocupado com o desenvolvimento pessoal, em vez de se preocupar com os limites do conhecimento dentro do campo mais amplo da profissão. Em contrapartida, a pesquisa de um doutorado em arquitetura deve ser um inquérito sistemático, o que significa ser destinado a produzir resultados confiáveis que estejam sujeitos a interrogações e revisão crítica, e que contribuam para um corpo comum de conhecimento (BIGGS, 2003). Preparar os alunos de pós-graduação para essas tarefas deve ser o objetivo das pedagogias adotadas no ensino superior, e depende essencialmente da qualidade do corpo docente, dos alunos, da atividade de orientação e da infraestrutura disponível.

Nos últimos dez anos, várias pesquisas foram conduzidas questionando a pedagogia aplicada em programas de pós-graduação. Nos cursos da área de arquitetura e urbanismo há um "agravante": tal área é muito heterogênea, constituindo muitas vezes um microcosmo na universidade como um todo (GRIFFITH, 2007). Os estudos sobre as pedagogias apropriadas questionam a eficácia do nexo entre ensino e pesquisa, tão aplaudido e defendido no ensino superior. A Arquitetura é uma área aplicada que abrange questões tanto tecnológicas quanto socioculturais. Nesse ambiente, a identidade acadêmica é mais fraca: as pesquisas da área procuram aplicar conhecimento para resolver problemas reais no ambiente construído, criar políticas ou apoiar a profissão nas suas atividades.

Os resultados dos referidos estudos mostram que os cursos de pósgraduação nem sempre preparam os alunos para as suas atividades de pesquisa. Dessa maneira, é necessário um novo olhar sobre o ensino na pósgraduação. $\mathrm{O}$ ensino clássico visa à transmissão da informação com base no conhecimento do professor e na ementa da disciplina. Os seminários, presentes na maioria dos melhores programas de pós-graduação em áreas das ciências exatas, biológicas e sociais, têm principalmente esse papel (GREEN; LEE, 1995). Outro tipo de ensino, mais apropriado para as áreas aplicadas e multidisciplinares, é o processo de aprendizagem baseado no aluno. Nesse tipo de ensino, o aluno possui um papel ativo e 0 objetivo não é necessariamente o aumento de conhecimento inédito, mas a transformação do aluno em um investigador independente (GRIFFITH, 2007). A elaboração dos tradicionais seminários passa a ser encargo dos 
alunos. Na maioria das vezes, esses seminários substituem a transmissão de conhecimento do professor para o aluno ou grupo de alunos, sem uma visão mais clara, abrangente ou focada do assunto em pauta. As dinâmicas de ensino aplicadas apresentadas nesse trabalho foram criadas para contornar tais fraquezas. Tendo por objetivo principal promover o incremento das pedagogias atuais e dar aos alunos maiores oportunidades de desenvolver, gerenciar e demonstrar suas pesquisas, as dinâmicas também contribuem na melhoria do ensino de projeto em outros níveis, tais como em cursos de graduação e extensão.

\section{DINÂMICAS DE ENSINO}

O ensino sobre o processo de projeto e principalmente sobre a fase analítica desse processo (programa arquitetônico) acontece em várias disciplinas. Em disciplinas de projeto do curso de graduação em Arquitetura e Urbanismo da Unicamp, por exemplo, várias dinâmicas são aplicadas na fase do pré-projeto.

\section{Ensino de graduação}

No ensino de graduação, foram introduzidas as experiências detalhadas a seguir:

\section{Brainstorming aplicado ao tema de interesse da disciplina, segundo as seguintes recomendações metodológicas:}

O intuito da técnica do brainstorming é desenvolver o máximo possível de soluções, que seriam examinadas e selecionadas. Osborn (1957) criou quatro regras básicas a ser observadas:

a) Sem críticas: a produção de ideias deve ocorrer num clima de liberdade;

b) Quantidade: a meta é atingir um grande número de ideias;

c) Geração em cadeia: as ideias iniciais servem como estímulo para as próximas;

d) Mutação e combinação: distorcer ideias ou combiná-las a outras ajuda a manter o fluxo de ideias dos participantes.

Apesar de considerado uma técnica a ser aplicada em grupo, o brainstorming também pode ser aposto a uma reflexão individual. Quando aplicado individualmente, tende-se a gerar ideias mais livres e explorar mais campos, pois o receio em relação a críticas é menor. Já em grupo, as ideias costumam ser mais eficientes devido à experiência e à diversidade dos participantes.

Para Davis (1992), Osborn percebeu, mesmo sem o estudo de métodos, que o indivíduo não pode ser ao mesmo tempo criativo e crítico. Em sua concepção, a separação em duas fases ajuda a distinguir esses dois tipos de raciocínio; há a divergência e a convergência. A primeira fase, essencialmente divergente, recorre à associação de ideias, permitindo ao participante expressar-se sem interrupção e criar vínculos entre seus pensamentos e os de outros. A segunda fase estimula o pensamento convergente na busca pela hierarquização das sugestões, de modo a extrair as que melhor se adequam ao problema em questão.

O uso do brainstorming pode ser aplicado a várias instâncias da atividade de ateliê de projeto, além de incluir grupos pequenos ou a classe toda na dinâmica. Em um primeiro momento, a prática de brainstorming se apresenta como um modo de estruturar as primeiras ideias e dúvidas 
do projeto, da organização do programa ou da definição do problema. Em seguida, a relação de termos e conceitos elencados é analisada sob outros critérios, o que oferece ao aluno alguns objetivos projetuais melhor definidos. Outra contribuição metodológica do brainstorming no processo de projeto é propiciar um momento de início das atividades, no qual o aluno supera a primeira inibição e se envolve na tarefa proposta.

\section{Jogo de cartas de arquitetura escolar e jogo DCBA Sustainable Game:}

O desenvolvimento de jogos conceituais de projeto é uma abordagem promissora para oferecer suporte aos diferentes agentes nos processos colaborativos. As razões apresentadas pela literatura para justificar os jogos nos processos de projeto são:

a) auxiliar os projetistas na tarefa de projetar edifícios complexos;

b) propor sistemas capazes de organizar a participação dos envolvidos, com a principal vantagem de permitir a criação de um cenário comum, em que todos podem se relacionar;

c) possibilitar o aprofundamento dos aspectos mais relevantes dos projetos, considerando os diversos tipos de usuários envolvidos;

d) elaborar as questões primordiais e seu grau de importância para a busca de soluções mais adequadas.

Um benefício da utilização dessa técnica é que ela demanda conhecimento e estudo da ferramenta, mas não alguma habilidade específica para sua utilização. Aumenta-se a compreensão do processo de planejamento e de sua complexidade e, consequentemente, o compromisso dos envolvidos. Também se obtém mais facilmente o consenso em arranjos básicos, mais do que com métodos tradicionais de busca de soluções. Os jogos exploratórios de projeto podem engajar e divertir as pessoas, criando uma atmosfera informal bastante produtiva no campo da criatividade (BRANDT, 2006; BRANDT; MESSETER, 2004).

As definições de projeto desses jogos dependem do escopo a ser atingido, de quem são os participantes envolvidos e também dos recursos disponíveis. Nas disciplinas de projeto do curso de graduação foram testados dois jogos: jogo de cartas de arquitetura escolar e o jogo DCBA. O primeiro foi aplicado para familiarizar os alunos com os conceitos e questões do ambiente escolar e estimular o debate para assim desenvolver um programa arquitetônico em comum dos integrantes da disciplina do semestre (Figura 1). O segundo jogo DCBA foi um jogo desenvolvido na Holanda para a definição de indicadores de sustentabilidade para projetos de arquitetura. O resultado da aplicação da dinâmica com base em jogos foi positivo, ainda que o jogo DCBA exija grande contribuição dos docentes em relação ao conhecimento específico e científico e que os alunos ainda não dominam. Deve-se testar a aplicação desse jogo ainda outras vezes com alunos novatos e com os alunos já mais maduros, possuintes do conhecimento do conceito da construção sustentável.

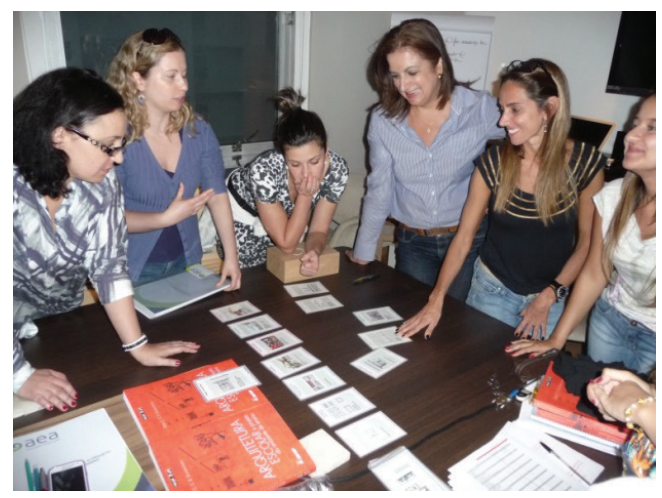

Figura 1. Aplicação de jogo de cartas para a programação de ambiente escolar, exemplo de dinâmica de ensino. Fonte: Doris Kowaltowski. 


\section{Pergunta e resposta em seminários:}

Essa dinâmica foi introduzida para tirar maior proveito dos clássicos seminários apresentados em disciplinas por alunos e permitir que os alunos interajam com os colegas. Embora haja uma preparação para a prática do seminário, ela ainda é pouca. Sendo assim, tanto na condução da atividade como na descrição do conteúdo as apresentações dos alunos de graduação não são suficientes para engajar os colegas nas discussões. Observou-se, portanto, a necessidade de introduzir um método que envolvesse todo o grupo de alunos, tanto nos momentos de apresentação como de audiência. Em disciplinas de ateliê deve ser dada especial atenção à transição do conteúdo do seminário para a prática de projeto, educando o aluno na atividade analítica de projeto.

Para esses objetivos foram desenvolvidas fichas com perguntas para os alunos da audiência e também para os membros do grupo que ministra o seminário. Essa dinâmica aumentou a atenção dos ouvintes e os apresentadores focaram mais em seus próprios objetivos. Além disso, criouse a oportunidade de introduzir a literatura sobre análise de projeto com a apresentação dos títulos Design strategies in architecture (BAKER, 1996), Analysing architecture (UNWIN, 1997) e Arquitetura: forma, espaço e ordem (CHING, 2013).

\section{Avaliação - dificuldades e facilidades:}

Essa dinâmica foi aplicada a várias disciplinas de projeto do curso de graduação em arquitetura e urbanismo no momento da entrega dos resultados das avaliações dos projetos desenvolvidos pelos alunos no semestre, com anotações de correções. Nessa atividade, é dada a oportunidade de expressão e manifestação, tanto aos docentes como aos alunos, em um espaço igual e comum na lousa da sala de aula. Dessa maneira, surgem pontos positivos e negativos, desafios e frustrações. Como resultado, é possível redirecionar as atividades didáticas do semestre e reforçar conceitos considerados importantes por ambos, alunos e docentes. Os alunos percebem muitas vezes, pela primeira vez, os objetivos de uma disciplina de projeto: o desenvolvimento do processo de projeto. A contribuição fundamental dessa dinâmica é uma confiança recíproca entre alunos e professores, especialmente se aplicada sem a atribuição de nota.

\section{Ensino de pós-graduação}

No programa de pós-graduação em ATC da Unicamp foram introduzidas as dinâmicas a seguir:

1) Na disciplina "AQ036 - Ambiente construído e comportamento humano”, as dinâmicas aplicadas foram estruturadas sobre os conceitos da obra A pattern language, de Alexander et al. (1977). A importância do conceito dos patterns foi abordada na disciplina, uma vez que a obra de Alexander oferece uma estrutura conceitual para enunciar parâmetros de projeto. Além de descrições precisas dos aspectos envolvidos na qualidade do projeto, a obra oferece uma metodologia para a condução do processo de projeto ao relacionar os padrões uns com os outros e abordar diferentes escalas de atuação. Também é um aspecto fundamental na obra de Alexander o fato de que os patterns apresentam conceitos de humanização em arquitetura. Esse conceito de humanização tanto instiga o interesse dos alunos na obra - ao aproximar as discussões de projeto às questões mais próximas do modo de vida e do comportamento do ser humano - como é tomado pelos críticos de Alexander como um aspecto 
questionável, associando a linguagem arquitetônica com soluções vernáculas e subjetivas. Dessa maneira, as dinâmicas baseadas nos patterns têm como objetivo familiarizar os alunos com a metodologia, pois, no Brasil, essa obra não integra a bibliografia básica da maioria das disciplinas de projeto nas escolas de arquitetura. A seguir são apresentados os detalhamentos de dois exercícios desenvolvidos na forma de dinâmicas e que envolviam todos os alunos da disciplina:

a) Desenvolver um novo pattern (parâmetro de projeto):

Na dinâmica da Figura 2, um grupo de alunos propôs o desenvolvimento de um parque a partir do conceito básico do pattern: "se existe uma situação que necessita de intervenção para tornar o ambiente mais humanizado, então o lugar deve demonstrar a sua potencialidade de intervenções físicas”. Foi utilizada a analogia de um quebra cabeça apresentado na Figura 2. A última peça e seu significado (pattern) foi uma decisão coletiva dos alunos da disciplina.

b) Identificar patterns na análise de projetos:

Essa dinâmica consiste na identificação de parâmetros de projetos apresentados por uma determinada equipe aos demais alunos da disciplina. o objetivo era demonstrar o poder dos patterns na sua visualização em plantas, cortes e imagens de projetos. A dinâmica também buscou envolver os alunos no reconhecimento dos patterns a partir de uma imagem retirada da obra de Alexander et al. (1977) (Figura 3). Na aplicação da dinâmica foi usado um dado (octaedro) com imagens originais dos próprios patterns. Como resultado, o grupo de alunos não somente identificou o pattern original como também fixou o conteúdo "se - então" do conceito dos patterns. Assim, o aprendizado dos participantes da disciplina foi testado e aprofundado.

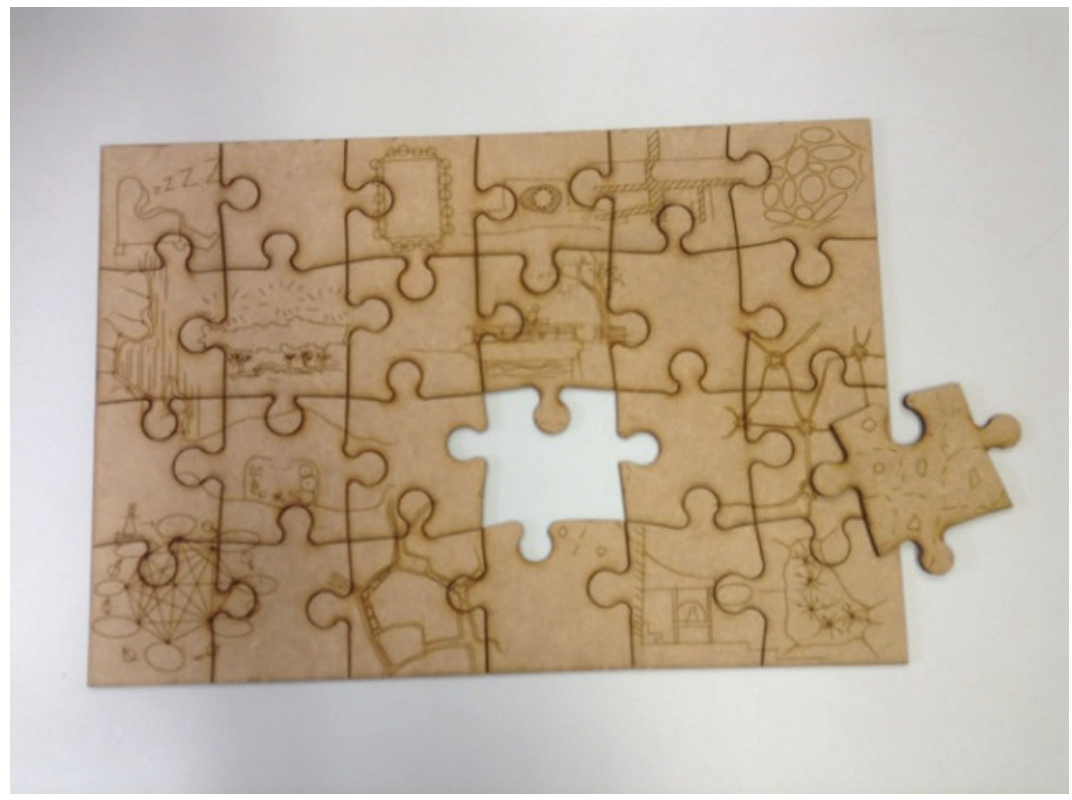

Figura 2. Exemplo de dinâmica introduzida por alunos em disciplina sobre o livro $A$ pattern language, de Alexander et al. (1977): quebra-cabeça. Fonte: Doris Kowaltowski. 
Figura 3. Exemplo de dinâmica introduzida por alunos em disciplina sobre o livro $A$ pattern language, de Alexander et al. (1977): octaedro. Fonte: Doris Kowaltowski.

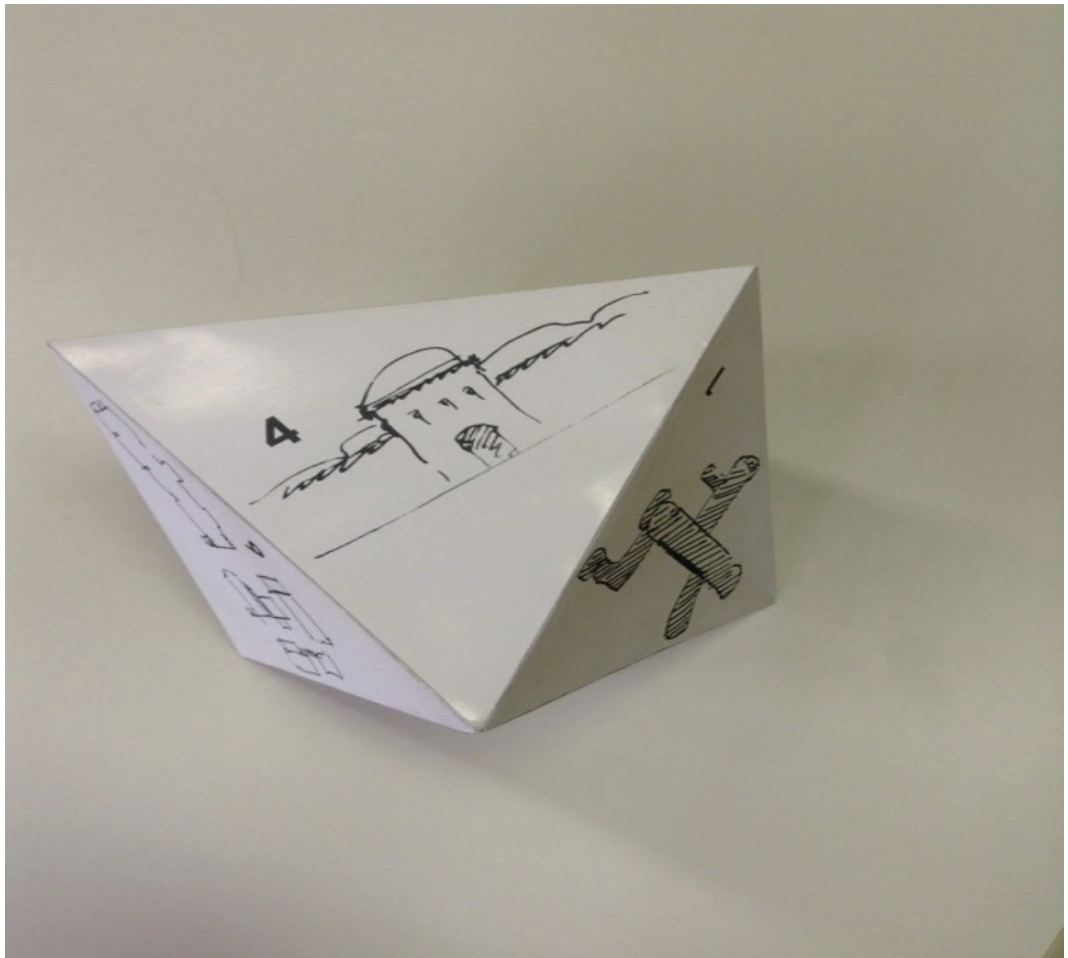

2) Em outra turma da disciplina "AQ036 - Ambiente construído e comportamento humano", o debate sobre o conceito da psicologia ambiental, com ênfase na percepção, foi aprofundado com dinâmicas específicas que ampliam o conceito da humanização da arquitetura. Essa disciplina contava com alunos arquitetos-urbanistas e engenheiros civis, mecânicos e ambientais. As discussões promovidas por essas dinâmicas procuram destacar que a compreensão do projeto arquitetônico deve ser aprofundada além da forma, da funcionalidade ou da viabilidade construtiva. Assim, foram propostas as dinâmicas:

a) Debate e observação de dois grupos distintos:

Essa dinâmica procura demonstrar o comportamento humano a partir da identificação visual das pessoas usando aleatoriamente uma identificação (cor, chapéu etc.). Esse exercício é considerado importante para a condução com ética de processos participativos, com debates sobre os conceitos de preconceito e inclusão/exclusão.

b) Entrando no elevador:

Essa dinâmica procura demonstrar o conceito da densidade ou do comportamento humano perante diferentes níveis de aglomeração (crowding). A dinâmica consiste em um grupo de alunos observando outro grupo entrando em um espaço pequeno (analogia do elevador) e identificando tipos de alteração de comportamento. O impacto da densidade populacional é discutido ao final, com recomendações sobre o projeto de ambientes que recebem grandes lotações como shopping centers, estádios ou pátios de escola, por exemplo.

c) Lei de Weber-Fechner:

Essa dinâmica consiste em demonstrar a lei de Weber-Fechner (PINKER, 2012), que descreve a relação existente entre a magnitude física de um estímulo e a intensidade do estímulo; ela é percebida pelos exemplos reais (ruído de cinco secadores de cabelos ligados ao mesmo tempo incomodam 
igualmente a um secador ligado, por exemplo), sendo que a relação entre a intensidade do estímulo é logarítmica em relação a sua percepção.

d) Julgar imagens com escala semântica:

Essa dinâmica estuda a percepção humana em relação às imagens escolhidas especificamente. 0 estudo visa ampliar a percepção do projetista em relação aos elementos visuais de um ambiente (dimensões, luz, cor, estilo estético etc.). $\mathrm{O}$ estudo também é aplicado para medir o grau de homogeneidade das percepções em processos participativos de projeto.

e) Procura-se o arquiteto sensível:

O exercício do arquiteto sensível se foca numa síntese dos debates desenvolvidos na disciplina sobre psicologia ambiental. Os temas da psicologia ambiental apresentados foram: ambiente construído e determinismo, relação ambiente-comportamento humano, equilíbrio de estímulo, componentes do comportamento, antropometria, proxêmica, densidade (crowding), proxêmica, territorialidade, defesa/segurança, privacidade, percepção (sala de Ames), ilusão ótica, entasis, teoria de Brunswick, escala, affordance (teoria de Gibson), teoria de Berlyne, fenomenologia, Wertheimer (teoria de Gestalt), Arnheim (visual thinking), Lynch (legibilidade do ambiente construído), environmental awareness and numbness, lei de Weber-Fechner, percepção estética, mecanismos da percepção, sentidos sensoriais, cognição, significado, espaço sociopetal/ sociofugal e, finalmente, wayfinding. A partir dessa introdução abrangente de conceitos da psicologia ambiental, os alunos deveriam identificar um profissional arquiteto e a sua obra, relacionando elementos arquitetônicos aos conceitos específicos da psicologia ambiental e ainda demonstrar, na trajetória do arquiteto, a sua sensibilidade aos conceitos da psicologia ambiental.

3) Na disciplina "AQ072 - Metodologia do projeto arquitetônico", algumas dinâmicas foram criadas pelos alunos a partir de uma variedade bibliográfica associada à produção dos teóricos dos métodos de projeto. A disciplina procurou demonstrar a importância da adoção de protocolos no desenvolvimento de diferentes fases do processo de projeto. As dinâmicas demonstraram como os métodos identificados na literatura otimizam o processo de projeto e apoiam a qualidade das soluções:

a) Antigos e novos métodos de projeto na literatura:

A partir da definição do projeto sistemático proposta por Jones (1963) no início dos anos 1960, as fases de análise, síntese e avaliação são interpretadas atualmente como procedimentos de divergência, transformação e convergência. Os procedimentos de convergência incluem a busca por limites, checklists e especificação escrita; os de transformação são exemplificados pelo brainstorming e interação matricial; já os procedimentos de convergência têm como exemplos a pesquisa na literatura e também o brainstorming. A dinâmica proposta na discussão sobre os antigos e novos métodos de projeto foi identificar procedimentos para uma variedade de problemas de projeto, como "quais soluções são importantes para o usuário em um projeto de habitação” ou "analisar as interações desejáveis e indesejáveis entre fluxos e ambientes de um hospital", ou mesmo "como encontrar a espessura mínima para uma cadeira de polipropileno”.

b) Aplicação e desenvolvimento do programa arquitetônico:

A literatura sobre o programa arquitetônico é rica em exemplos de procedimentos complexos de levantamento de informações para o projeto aplicados ao cliente e ao usuário. A dinâmica proposta pelos alunos consistiu em experimentar o método de organização do programa 
arquitetônico segundo o Problem Seeking (PEÑA; PARSHALL, 2012). Foram distribuídos cartões entre as equipes de alunos que deveriam se concentrar na organização das metas, fatos, conceitos e necessidades para o projeto de uma residência para uma família específica, cujas características foram detalhadas no enunciado do exercício (bem como o contexto onde o projeto se desenvolveria) de tal modo que os problemas de projeto deveriam ser estabelecidos e discutidos pelo conjunto de alunos da disciplina. A organização e a ilustração das questões em cartões deram origem a um complexo conjunto de temas para discussão.

c) Programa e tipologias:

A definição do caráter funcional da edificação permite identificar uma literatura específica sobre determinada organização ou tipologia. Compreender a variação dos valores e conceitos de uma determinada tipologia ao longo da história oferece um recurso importante para o desenvolvimento de novos projetos, principalmente na etapa do programa arquitetônico: a capacidade de adaptação de uma construção segundo alterações de uso ao longo do tempo é uma variável de projeto de difícil trabalho. Para demostrar esse conceito, a dinâmica proposta pelos alunos recuperou a clássica questão de como seria projetar a primeira casa para o homem primitivo.

d) Novos métodos de criatividade:

O estímulo à criatividade é uma questão frequentemente retomada nas discussões sobre os métodos de projeto. É comum recuperar questões sobre a fisiologia e as habilidades de cada hemisfério cerebral, regras de conduta social, atividades físicas e mentais de estímulo, organização pessoal e até quais ambientes são mais favoráveis à concentração ou ao devaneio.

Cientes das implicações da criatividade no processo de projeto, os alunos foram convidados a apresentar dinâmicas de estímulo à solução de alguns problemas pontuais de projeto. Foram experimentados procedimentos da área do design do objeto baseados em restrições e condicionantes. Também foi proposto um exercício de projeto baseado em Role Playing Game (RPG), o que estimula os participantes a adotar uma linha de pensamento objetiva e fiel ao perfil do personagem assumido e otimiza as propostas. Embora algumas reações e propostas possam ser estereotipadas, é um aspecto importante da dinâmica a fluidez com que a participação, as ideias e o debate de projeto são colocados.

e) Procedimentos de análise e avaliação de projetos:

Essa dinâmica introduziu os conceitos de análise e avaliação de projetos, enfatizando as diferenças entre os dois procedimentos e apresentando métodos apropriados a cada um. Nessa dinâmica foram apresentados aos alunos dois projetos (somente os desenhos e croquis dos autores) e quatro métodos, dois de análise e dois de avaliação.

A análise de projetos contribui para a reunião de exemplos e soluções precedentes, selecionados para resolver determinado aspecto em discussão durante o processo de concepção de um novo edifício. Além da experiência no desenvolvimento de outros projetos, o arquiteto obtém repertório e referências ao estudar a forma, a estrutura ou a função de outros edifícios. Esse processo de análise é conduzido por meio de desenhos, esboços e diagramas, uma vez que a síntese gráfica permite destacar determinadas soluções e compará-las com outras, resultando em alternativas para novos projetos. A partir dessas propriedades, a dinâmica de análise propõe 0 exercício de identificação de aspectos compositivos em um determinado projeto. O exercício apresenta apenas os desenhos de dois projetos e uma lista de verificação desenvolvida segundo dois métodos de análise de projeto (BAKER, 1996; UNWIN, 1997). Os alunos, organizados em duplas, 
devem, para cada critério de análise, propor observações ou críticas sobre como cada aspecto foi introduzido ou resolvido no projeto. Como resultado, foi observado que confrontar as críticas de vários alunos potencializa a compreensão conjunta de um projeto, além de estimular discussões objetivas e criativas sobre vários aspectos de uma mesma edificação.

Projeto 1

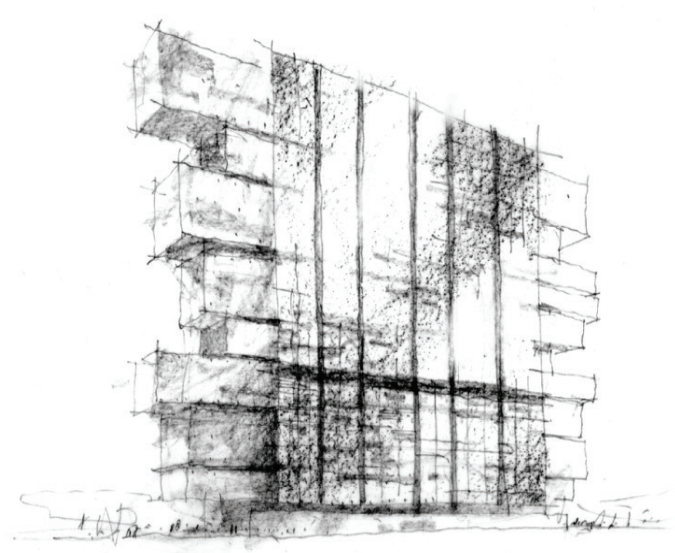

Manchester-civil-justice-centre, UK, Denton-CorkerMarshall Arch. (2008).
Projeto 2

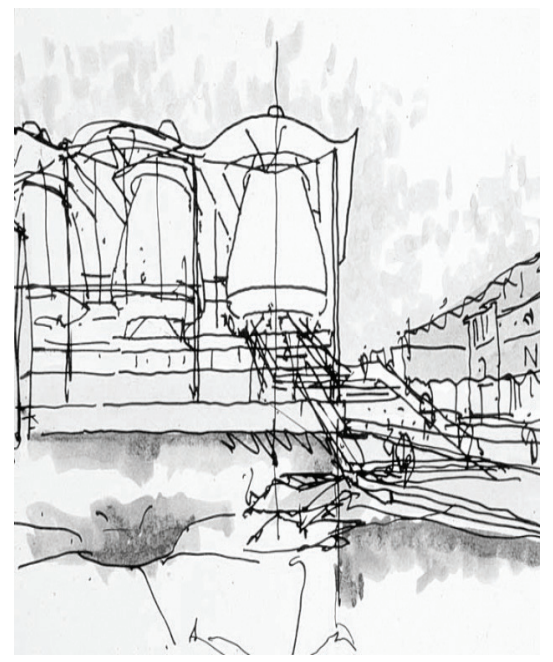

Bordeaux Law Courts, França, Richard Rogers Partnership (1998).
A avaliação de projetos consiste na adoção de determinados parâmetros, qualitativos e quantitativos, que permitem mensurar soluções de projeto segundo determinados critérios. O processo tende a isolar várias propriedades para a avaliação e considerar as soluções observadas segundo o grau de adaptação que a edificação demonstra em relação a um critério. Com frequência, a avaliação procura identificar desempenhos de ordem funcional, estrutural, compositiva, de infraestrutura urbana e de conforto. As dinâmicas de avaliação são importantes para familiarizar o aluno com a adoção de critérios mais precisos em projeto. Por isso, os exercícios de avaliação costumam adotar uma lista de fatores obtida na literatura - segundo uma tipologia funcional, um checklist de desempenho ou características universais de projeto - que é aplicada na avaliação de um projeto selecionado segundo a natureza do problema em discussão. Como no exercício de análise descrito anteriormente, dois procedimentos de avaliação foram selecionados na literatura (VAN DER VOORDT et al., 1997; WONG et al., 2009) e apresentados aos alunos da disciplina para comparação de dois projetos.

\section{CONCLUSÕES}

As dinâmicas proporcionam a assimilação de novas literaturas em metodologia de projeto e psicologia ambiental em arquitetura. Comprovou-se tal hipótese pela organização do conhecimento adquirido no desenvolvimento de práticas em sala de aula.

Nesse trabalho foram apresentados dois tipos de dinâmicas. Os docentes das disciplinas aplicaram um tipo de dinâmica no ateliê de projeto e em disciplinas de pós-graduação, e o outro tipo foi desenvolvido pelos alunos para envolver diretamente o grupo todo. O primeiro tipo compreendeu as dinâmicas: brainstorming, jogos de cartas, perguntas e respostas em
Figura 4. Projetos de arquitetura apresentados na dinâmica de análise e avaliação. 
seminários, avaliação - dificuldades e facilidades, debate e observação de dois grupos distintos, entrando no elevador, lei de Weber-Fechner, julgar imagens com escala semântica, antigos e novos métodos de projeto na literatura, procedimentos de análise e avaliação de projetos. O segundo tipo, desenvolvido pelos alunos, incluiu as dinâmicas: desenvolver um novo pattern, identificar patterns na análise de projetos, procura-se o arquiteto sensível, aplicação e desenvolvimento do programa arquitetônico e programa e tipologias.

As lições aprendidas dessas experiências de dinâmicas de ensino em metodologia de projeto foram variadas, com respostas positivas e resultados que merecem maior atenção em sua pedagogia. Mesmo com boa qualidade, as dinâmicas na pós-graduação não conseguiram ser apresentadas ou concluídas como artigos científicos para uma crítica científica de pares. A contribuição para o ensino de pós-graduação necessita de avaliação, comprovação e divulgação - como aventado por esse artigo. Na graduação, as dinâmicas envolvem os alunos em atividades que resultam em importantes descobertas sobre as implicações do projeto em desenvolvimento, mas que, com frequência, também demonstram a pouca compreensão, por parte do aluno, de conceitos de desenho e da teoria da arquitetura. Para que as dinâmicas tenham sucesso, o professor deve entender o aluno de graduação e de pós-graduação, segundo seus estilos de aprendizagem, seus talentos e as habilidades da Geração Y.

É importante considerar uma introdução sobre o tema por trás da dinâmica a ser desenvolvida, apresentada pelos docentes. Assim, as supostas brincadeiras envolvidas nas atividades exigem, na verdade, preparação e atenção na aplicação. Uma estratégia é o estudo de referências científicas para definição dos objetivos das dinâmicas desenvolvidas. Nas disciplinas de pós-graduação, onde os alunos são incentivados a desenvolver dinâmicas a partir de uma fundamentação científica, é possível dar liberdade ao escolher o tipo de dinâmica, sua apresentação, seu método, objetivo e resultado alcançado. Como resposta, as dinâmicas desenvolvidas pelos alunos são, em muitos casos, surpreendentes e excelentes. As experiências relatadas neste artigo demonstram que o aluno se envolve de modo positivo nas atividades de dinâmicas, o que é resultado de um estímulo e de um incentivo além das tradicionais atividades de seminários. Espera-se que as questões levantadas aqui estimulem novas abordagens no ensino de projeto, para que o ateliê de arquitetura se desenvolva e acompanhe as grandes dinâmicas tecnológicas sem perder seu caráter e sempre atentar para as questões fundamentais e atemporais do bom projeto.

\section{REFERÊNCIAS}

AKÖZER, E. Doctorates in architecture: the bologna process and the critical/ humanist tradition. In: BELDERBOS, M.; DUJARDIN, M.; VERBEKE, J.; WOYSETH, H. D.; GENARD, J. L.; GLANVILLE, R.; HEYNEN, H.; VANDERBURGH, D.; VERSCHAFFEL, B. (Eds.). The unthinkable doctorate. Cidade de Bruxelas: Hogeschool voor Wetenschap en Kunst, 2005. p. 141-150.

ALEXANDER, C.; ISHIKAWA, S.; SILVERSTEIN, M. A pattern language: towns, buildings, construction. Cambridge: Oxford University Press, 1977.

BAKER, G. H. Design strategies in architecture: an approach to the analysis of form. Nova York: Van Nostrand Reinhold; E \& F N Spon, 1996.
BAYAZIT, N. Investigating design: a review of forty years of design research. Design Issues, Nova York, v. 20, n. 1, p. 16-29, 2004.

BIGGS, M. A. R. The role of "the work" in research. In: PRACTICE AS RESEARCH IN PERFORMANCE, 2003, Bristol. Proceedings... Bristol: University of Bristol, 2003. Disponível em: <http://www.bris. ac.uk/parip/biggs.htm>. Acesso em: 27 jun. 2014.

BRANDT, E. Designing exploratory design games: a framework for participation in participatory design? In: CONFERENCE ON PARTICIPATORY DESIGN, 9., 2006, Nova York. Anais... Nova York: ACM, 2006. p. 5766. 
BRANDT, E.; MESSETER, J. Facilitating collaboration through design games. In: CONFERENCE ON PARTICIPATORY DESIGN, 8., 2004, Nova York. Anais... Nova York: ACM, 2004. p. 121-131.

BROADBENT, G.; WARD, A. Design methods in architecture. Nova York: Lund Humphries, 1969.

BUCHANAN, R. Wicked problems in design thinking. Design Issues, Nova York, v. 8, n. 2 p. 5-21, 1992. DOI: 10.2307/1511637.

CHING, F. D. K. Arquitetura: forma, espaço e ordem. 3. ed. Porto Alegre: Bookman, 2013.

CROSS, N. (Ed.). Developments in design methodology. Nova Jersey: Wiley, 1984.

CROSS, N. Designerly ways of knowing Berlim: Springer, 2006.

DAVIS, G. A. Creativity is forever. 3. ed. Dubuque: Kendall; Hunt Pub, 1992.

DOGAN, F.; ZIMRING, C. Interaction of programming and design: the first unitarian congregation of Rochester and Louis I. Kahn. Journal of Architectural Education, v. 56, n. 1, p. 47-56, 2002. DOI: 10.1162/104648802321019164.

ESHERICK, J.; HASSID, S.; MOORE, C. Graduate programs 1: The University of California. Journal of Architectural Education, v. 18, n. 2, p. 21-24, 1963.

FRAMPTON, K. Modern architecture: a critical history. 4. ed. Nova York: Thames \& Hudson, 2007

GRIFFITHS, R. Knowledge production and the research-teaching nexus: the case of the built environment disciplines. Studies in Higher Education, v. 29, n. 6, p. 709-726 2004. DOI: 10.1080/0307507042000287212.

HERSHBERGER, R. G. Architectural programming \& predesign manager. Nova York: McGraw-Hill, 1999.

KOWALTOWSKI, D. C. C. K. Metodologia e CAD no Projeto Arquitetônico. In: SEMINÁRIO INTERNACIONAL FAUUSP - COMPUTAÇÃO: ARQUITETURA E URBANISMO, 1992, São Paulo. Anais... São Paulo: FAU-USP, 1992. p. 51-57.

KOWALTOWSKI, D. C. C. K.; CELANI, M. G. C.; MOREIRA, D. C.; PINA, S. A. M. G.; RUSCHEL, R. C.; SILVA, V. G.; LABAKI, L. C.; PETRECHE, J. R. D. Reflexão sobre metodologias de projeto arquitetônico. Ambiente Construído, v. 6, n. 2, p. 7-19, 2006.

JONES, J. C. A method of systematic design In: JONES, J. C.; THORNLEY, D. G. (Eds.). Conference on design methods. Oxford: Pergamon Press, 1963.
KUMLIN, R. Architectural programming creative techniques for design professionals. Nova York: McGraw-Hill, 1995.

LAMPRECHT, B. Richard Neutra, 18921970: survival through design. Colônia: Taschen, 2009.

MOREIRA, D. C.; KOWALTOWSKI, D. C. C. K. Discussão sobre a importância do programa de necessidades para a qualidade no processo de projeto em arquitetura. Ambiente Construído, v. 9, n. 2, p. 31-45, 2009 .

ORNSTEIN, S. W.; ROMÉRO, M. Avaliação pós-ocupação do ambiente construído São Paulo: Studio Nobel, 1992

OSBORN, A. F. Applied imagination: principles and procedures of creative problem-solving. Nova York: Charles Scribner's Sons, 1957.

PALTRIDGE, B. Thesis and dissertation writing: an examination of published advice and actual practice. English for Specific Purposes, v. 21, n. 2, p 125-143, 2002. DOI: 10.1016/S08894906(00)00025-9

PEÑA, W. M.; PARSHALL, S. A. Problem seeking: an architectural programming primer. Nova York: Wiley, 2012.

PINKER, S. Better angels of our nature Londres: Penguin Books, 2012.

PREISER, W. F. E.; RABINOWITZ, H. Z.; WHITE, E. T. Post-occupancy evaluation. Nova York: Van Nostrand Reinhold, 1990

RITTEL, Horst. The State of the Art in Design Methods. Design Research and Methods (Design Methods and Theories). v. 7, n. 2 , p. 143-147, 1973.

ROWE, P. G. Design thinking. Cambridge: The MIT Press, 1991

UNWIN, S. Analysing architecture. Londres: Routledge, 1997

VAN DER VOORDT, D. J. M.; VRIELINK, D.; VAN WEGEN, H. B. R. Comparative floorplan-analysis in programming and architectural design. Design Studies, v. 18, n. 1, p. 67-88, 1997.

VAN DER VOORDT, D. J. M.; VAN WEGEN, $H$. B. R. Architecture in use: an introduction to the programming, design and evaluation of buildings. Oxford: Architectural Press Elsevier, 2005.

WONG, F. W. H.; LAM, P. T. I.; CHAN, E. Optimising design objectives using the Balanced Scorecard approach. Design Studies, v. 30, n. 4, p. 369-392, 2009. DOl: 10.1016/j.destud.2008.10.004
Daniel de Carvalho Moreira damore@fec.unicamp.br

Doris Catharine Cornelie Knatz Kowaltowski

doris@fec.unicamp.br

Renata Maria Geraldin Beltramin

renelens05@gmail.com 